ЛЕВИТАЦИЯ ПОСТОЯННОГО МАГНИТА В ОБЛАСТИ С ДИАМАГНИТНЫМИ ГРАНИЦАМИ ПРИ ВЕРТИКАЛЬНОЙ И ГОРИЗОНТАЛЬНОЙ ОРИЕНТАЦИИ МАГНИТНОГО ПОЛЯ

\author{
М. П. Волков
}

Физико-технический институт им. А. Ф.Иоффе РАН (Санкт-Петербург, Россия)

\author{
PERMANENT MAGNET LEVITATION IN A \\ REGION WITH DIAMAGNETIC BOUNDARIES \\ WITH A VERTICAL AND HORIZONTAL \\ ORIENTATION OF A MAGNETIC FIELD \\ M. P. Volkov \\ Ioffe Physico-Technical Institute \\ Russian Academy of Sciences \\ (St. Petersburg, Russia)
}

Пассивная левитация тел в постоянном магнитном поле может быть реализована с использованием явления диамагнетизма двумя способами: а) левитация диамагнитного тела в сильном неоднородном магнитном поле и б) левитация постоянного магнита в неоднородном магнитном поле при наличии расположенных рядом с магнитом массивных диамагнитных тел.

Для реализации первого способа требуются сильные магнитные поля (создаваемые, например, с помощью сверхпроводящих соленоидов), а второй способ может быть осуществлен при использовании относительно небольших магнитных полей, созданных системой постоянных магнитов. Именно этот способ привлекает большое внимание из-за возможных применений в технических системах, где требуется исключить связанную с трением диссипацию энергии, таких, как магнитные микроподшипники, чувствительные механические датчики, устройства преобразования механических колебаний в электрическую энергию и другие. 
Для магнитной левитации постоянного магнита можно использовать вертикально направленное магнитное поле системы постоянных магнитов при горизонтальном расположении стабилизирующих массивных диамагнитных пластин. Возможна и другая конфигурация - с горизонтально направленным магнитным полем и, соответственно, с вертикальным расположением диамагнитных пластин. В конфигурации с вертикальным магнитным полем подвешенный магнит может свободно перемещаться в горизонтальной плоскости на некоторое расстояние, и наоборот, при горизонтально направленным магнитным полем - в вертикальной плоскости. Выбор конкретной конфигурации магнитов определяется той технической задачей, для решения которой будет использоваться бесконтактная подвеска постоянного магнита.

В нашей работе проведено сравнение двух конфигураций - с вертикальным и горизонтальным магнитным полем - и показано, что оба варианта левитации магнита достигаются в одной и той же системе постоянных магнитов и диамагнитных пластин при разных ориентациях системы. В качестве стабилизирующих диамагнитных пластин использовались чистый висмут, технический графит и ориентированный пиролитический графит. Сделана оценка области стабильности при отклонении магнита от положения равновесия для обеих конфигураций.

\section{Information of authors:}

ВОЛКОВ Михаил Павлович, m.volkov@mail.ioffe.ru

Mikhail P. VOLKOV,m.volkov@mail.ioffe.ru 